\title{
Supporting Information for: On the (non)Existence of Bulk Nanobubbles: The Role of Ultrasonic Cavitation and Organic Solutes in Water
}

\author{
Dmytro Rak, Michaela Ovadová, and Marián Sedlák*
}

Institute of Experimental Physics, Slovak Academy of Sciences, Watsonova 47, 04001 Košice, Slovakia.

* corresponding author. Email: marsed@ saske.sk, tel.: +421 557922245

\section{Experimental methods and materials}

\author{
Materials \\ Water was purified by reversed osmosis and activated carbon TOC reduction, freshly double- \\ distilled in a quartz apparatus, and subsequently deionized by analytical grade mixed-bed ion \\ exchange resins (Bio-Rad, Richmond, CA). We have used also water from commercial apparatus \\ ELGA Purelab Ultra Analytic (Elga, United Kingdom). No dependence of results on water \\ source was observed. The resistivity of water was always above $18 \mathrm{M} \Omega \mathrm{cm}$. "Unpurified ethanol" \\ was from Centralchem (p.a. grade, $99.5 \%$ purity) and used as delivered. "Purified ethanol” was \\ obtained using the procedure based on our know-how gained in our previous work. ${ }^{1} 20 \%$ water \\ solution of the unpurified ethanol was filtered via nanoporous filters to remove practically \\ completely hydrophobic impurities segregated into nanodroplets. Ethanol was then distilled off \\ and water residuals were subsequently removed via drying over a molecular sieve. Full removal \\ of hydrophobic impurities was verified via HS-SPME (Head Space Solid-Phase Microextraction) \\ followed by GCMS (Gas Chromatography coupled with Mass Spectrometry) analysis.
}




\section{Ultrasound treatment}

Ultrasound treatment of water and ethanol-water mixtures were performed using Bandelin SONOPULS HD 3100 (Bandelin Electronic GmbH \& Co. KG, Germany) ultrasonic homogenizer. $25 \mathrm{~mL}$ of water or of ethanol-water mixture were poured in a round-bottom glass flask and sonicated using titanium probe (material no. 3.7165, Ti6Al4V) with $6 \mathrm{~mm}$ tip diameter (KE76). Amplitude of ultrasound with fixed frequency of $20 \mathrm{kHz}$ was set to $10 \%$ of its maximum value (equivalent to $12 \mathrm{~W}$ sonication power). Sonication time varied from 10 min to 60 min. Water and ethanol-water were filtered through $0.2 \mu \mathrm{m}$ filters prior to sonication.

\section{Incremental centrifugation coupled with light scattering (IC-LS)}

The principles of incremental centrifugation coupled with light scattering are described in detail in the main text. Samples were centrifuged in a microchip-controlled centrifuge Jouan KR22i (Jouan, France). This centrifuge enables very precisely to set not only the relative centrifugal force $(R C F)$ and centrifugation time $t$, but also the product $R C F t$ including the acceleration and braking by definition of the integral of the dependence of $R C F$ on $t$. This allows applying exactly and reproducibly centrifugal gravitational separation. Temperature was controlled during centrifugation by cooling. A swing-out rotor (SWK100.13, Jouan, France) was used in which the scattering cell is positioned perpendicular to the axis of rotation during centrifugation so that the gravitational force acts along the cell axis towards its bottom (see Figure S1). Light scattering was measured immediately before and after centrifugation. No kinetic changes were observed during the time necessary to perform light scattering measurement after careful removing the cell from the centrifuge. Static light scattering (SLS) measurements were made using a $40 \mathrm{~mW}$ HeNe laser, model 25LHP928 (CVI Melles Griot, Albuquerque, NM) with $632.8 \mathrm{~nm}$ vertically polarized beam and a laboratory made goniometer with angular range from $30^{\circ}$ to $135^{\circ}$. Scattering intensities were measured by photon counting. Dynamic light scattering (DLS) were made using the same optico-mechanical setup as SLS and ALV7004 correlator (ALV, Langen, Germany). Correlation curves were fitted by CONTIN program. ${ }^{2}$ A test example of an experiment of incremental centrifugation coupled with light scattering on a well-defined silica nanoparticles is shown in Fig. S2. 


\section{Calculation of density of nanoparticles}

Migration of a particle in a centrifuge tube from a position characterized by the distance $r_{1}$ from the axis of rotation to the position characterized by the distance $r_{2}$ is described by the sedimentation equation

$$
\ln \frac{r_{2}}{r_{1}}=\frac{2 R^{2} \Delta \rho}{9 \eta} \omega^{2} t
$$

where $R$ is the particle radius, $\Delta \rho$ is the difference between the density of the particle and the density of the surrounding liquid, $\eta$ is the dispersion viscosity, $\omega$ is the angular frequency of the rotor, and $t$ is the duration of centrifugation. The term $\left(2 R^{2} \Delta \rho\right) / 9 \eta$ is also known as the sedimentation coefficient. Instead of the rotor angular frequency $\omega$, centrifugal acceleration or relative centrifugal force $(R C F)$ is used in practice, where

$$
R C F=r \omega^{2}
$$

$r$ being the distance between the axis of rotation and the particle experiencing the given $R C F$. $R C F$ is conveniently expressed in units of Earth's gravitational acceleration $g$. As can be seen from eq. $\mathrm{S} 1$, a certain product of $R C F$ and time $t$ is necessary to translocate the particle over the desired distance $\left|r_{1}-r_{2}\right|$. This is because the particle moves with a constant terminal velocity, which is proportional to $R C F$. Referring to the scheme in the Fig. 1, $r_{1}$ is in our case the distance from the axis of rotation to the meniscus and $r_{2}$ is the distance from the axis of rotation to the position where the laser beam passes through the tube in a light scattering experiment (marked by a red line in Fig.1). The average density of particles can be then calculated from the value of the RCFt needed to move particles over the distance $\left|r_{1}-r_{2}\right|$, i.e. to remove particles from the probed scattering volume. $R C F t$ is taken in practice from the position of an inflection point in the dependence of scattering intensity on the applied $R C F t$, preferrably from the experiment with the longer $52 \mathrm{~mm}$ path. The particle radius $R$ entering the calculation is obtained from dynamic light scattering. While it is known that $R_{h}$ measured by DLS in polydisperse samples is dominated by the largest particles from the distribution, scattering intensity (Fig.1C, Fig.4A,B) is also dominated by the largest particles from the distribution. 


\section{Nanoparticle tracking analysis (NTA)}

NTA measurements were performed with an LM10B Nanoparticle characterization system from Nano Sight (Amesbury, U.K.) equipped with LM12 viewing unit with a $60 \mathrm{~mW}$ laser working at $\lambda=405 \mathrm{~nm}$. Video sequences were recorded via CCD camera operating at 30 frames per second (fps) and evaluated using NTA 3.1 Analytical Software Suite. All images shown in Fig. 3A-3D were acquired without any sample dilution. For acquiring videos and subsequent calculations of size distributions, samples shown in Figs 3B and 3D were diluted 300× to get optimum number of particles per frame for analysis of their diffusion tracks. Fig.S3 shows number-weighted size distributions of titanium nanoparticles calculated, complementing volume-weighted size distributions shown in Fig.2F in the main text.

\section{Scanning electron microscopy (SEM)}

Titanium particles for SEM analysis were concentrated ca. 8 times by centrifugation of $8 \mathrm{~mL}$ of the particle dispersion for 1 hour at relative centrifugal force (RCF) $20000 \mathrm{~g}$. Most of the particle-free supernatant was carefully removed after centrifugation and particles were resuspended in remaining amount of water by gentle shaking. One drop of the obtained dispersion was then placed on a carbon film coated copper mesh (Agar Scientific Ltd, United Kingdom) and left to dry in desiccator overnight. Dried sample was then placed on SEM specimen stub and fixed with conductive carbon tape. The morphology and elemental composition of nanoparticles were accessed using SEM equipped with focused ion beam (FIBSEM, Auriga Compact, Carl Zeiss, Germany) and energy dispersive spectrometer (EDS, Oxford Instruments, United Kingdom). Results from SEM and EDS are shown in Fig.S4.

\section{Inductively coupled plasma mass spectrometry (ICP-MS)}

Total concentrations of titanium and vanadium in samples were determined using ICP-MS preceded by microwave-assisted digestion. Samples containing nanoparticles were digested using the UltraWave single reaction chamber digestion unit (Milestone, Italy) with $3 \mathrm{~mL}$ of $\mathrm{HNO}_{3}$ according to the two-step temperature-controlled digestion program and diluted before the 
ICP-MS analysis. Measurements were performed on Agilent 7700x ORS-ICP-MS spectrometer (Agilent, Japan) fitted with a MicroMist concentric nebulizer, a Scott-type double-pass spray chamber, and octupole reaction system operating in helium mode to eliminate the possible spectral interferences. The monitored isotopes were ${ }^{46} \mathrm{Ti},{ }^{47} \mathrm{Ti},{ }^{48} \mathrm{Ti},{ }^{49} \mathrm{Ti},{ }^{50} \mathrm{Ti}$ for $\mathrm{Ti}$ and ${ }^{51} \mathrm{~V}$ for V. ${ }^{45} \mathrm{Sc}$ served as an internal standard. The two water CRMs having the certified values for Ti and $\mathrm{V}$ were repeatedly analyzed to assess the trueness and precision of measurements. Nitric acid used was 65\% Analpure ${ }^{\circledR}$ (Analytika Ltd., Czech Republic). Element certified reference material (CRM) at the concentration of $1000 \pm 2 \mathrm{mg} / \mathrm{L}(\mathrm{Ti}, \mathrm{V})$ or $10 \pm 0.1 \mathrm{mg} / \mathrm{L}$ INT-MIX 1 (Bi, In, Sc, Tb, Y) were from Analytika Ltd., Czech Republic. Ultrapure water was obtained from Milli-Q system (Millipore, France). Water certified reference materials used were TM 25.5 and TMDA 64.3 (Environment and Climate Change Canada, Canada).

\section{Spectrophotometric determination of titanium content}

Spectrophotometric determination of the concentration of $\mathrm{Ti}$ in samples was done in the form of a $\mathrm{Ti}(\mathrm{IV})-\mathrm{H}_{2} \mathrm{O}_{2}$ complex. $2 \mathrm{~mL}$ of $20 \%$ sulphuric acid was added to $10 \mathrm{~mL}$ of the sample to dissolve all of the titanium nanoparticles present in the sample. Solution was then boiled until its volume was reduced by half. Ti(III) in the obtained solution was then oxidized to Ti(IV) by addition of $0.2 \mathrm{~mL}$ of $\mathrm{HNO}_{3}$, and solution was again boiled to remove excess $\mathrm{HNO}_{3}$. Subsequently the solution was transferred to a $10 \mathrm{~mL}$ measuring flask containing $0.2 \mathrm{~mL}$ of the hydrogen peroxide (30\% solution) and the volume was adjusted with water. The optical density of the solution was then measured at $\lambda=420 \mathrm{~nm}$ and concentration of Ti alloy in the sample was determined using a calibration curve. The calibration curve was established using a standard solution prepared from alloy pieces cut from the ultrasonic probe actually used in experiments. Shimadzu UV-2600 UV-Vis spectrophotometer equipped with a $5 \mathrm{~cm}$ path length quartz cell was used for all optical density measurements. 


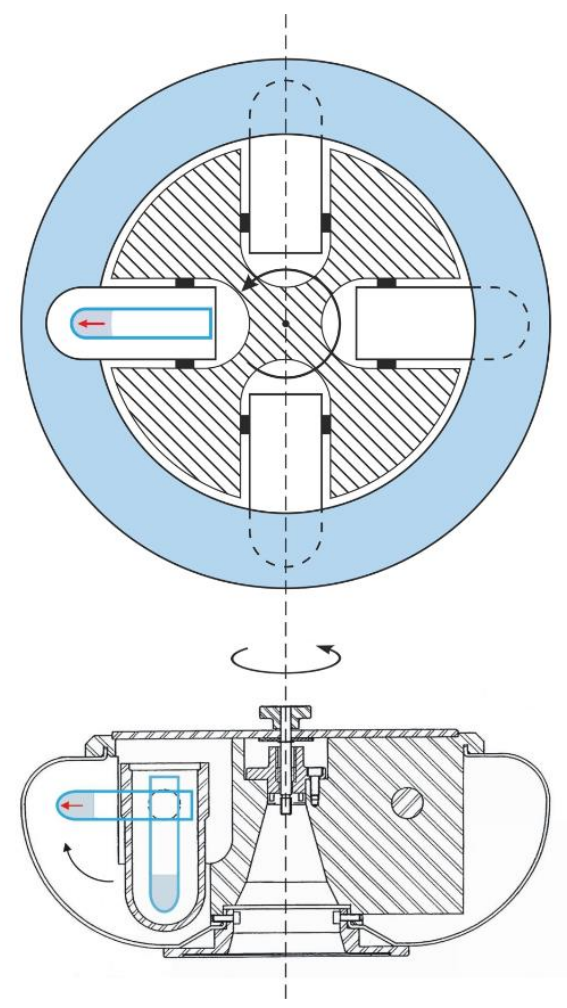

Fig. S1. Schematic representation of the swing rotor setup used in centrifugation experiments. Top crossectional view (above) and side crossectional view (below). 


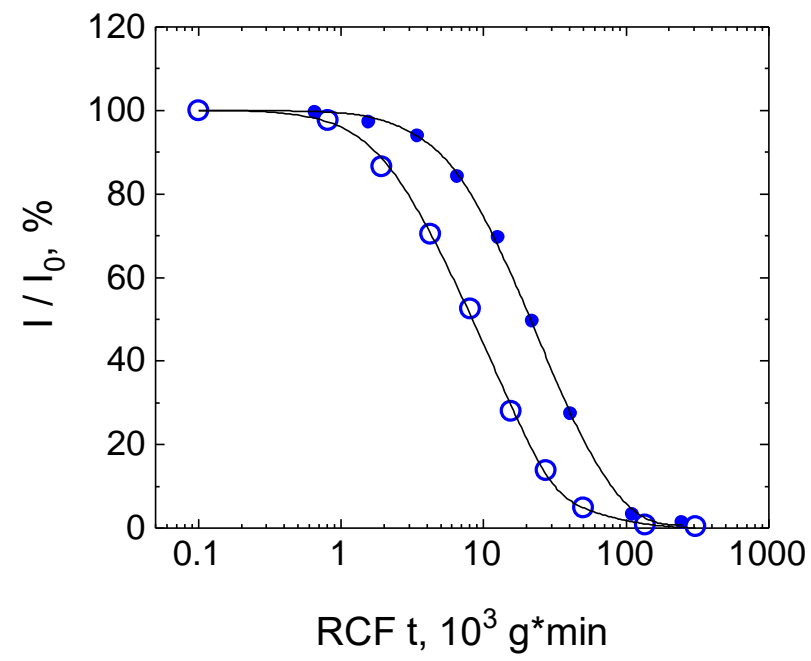

Fig. S2. Incremental centrifugation coupled with light scattering on silica beads with radius $\mathrm{R}=$ $140 \mathrm{~nm}$ (Bangs Laboratories, Inc., Fishers, IN, USA). Centrifugation tubes were filled with the solution such that distances from the meniscus to the position of the laser beam in a light

scattering setup were $6 \mathrm{~mm}(O)$ and $52 \mathrm{~mm}(\bullet)$, respectively. Scattering angle was $45^{\circ}$.

Calculated value of density of nanoparticles from data on the $52 \mathrm{~mm}$ path length: if the inflection point is taken as the point where $I / I_{0}=50 \%$, the density comes out as $\rho=1.87 \mathrm{~g} / \mathrm{cm}^{3}$. For $I / I_{0}=$ $45 \%$ the density comes out as $\rho=1.77 \mathrm{~g} / \mathrm{cm}^{3}$ and for $I / I_{0}=55 \%$, the density comes out as $\rho=$ $2.01 \mathrm{~g} / \mathrm{cm}^{3}$. The density provided by the manufacturer: $1.96 \mathrm{~g} / \mathrm{cm}^{3}$.

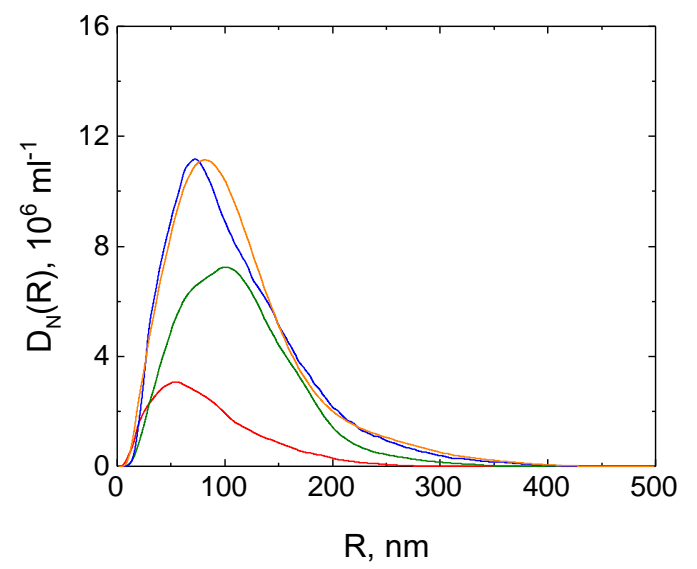

Fig. S3. Number-weighted size distributions of titanium nanoparticles calculated from NTA data presented in Fig.2F. Red, green, and blue curves correspond to the samples of water treated with ultrasound for $10 \mathrm{~min}, 25 \mathrm{~min}$, and $60 \mathrm{~min}$, respectively. Orange curve corresponds to the sample of degassed water sonicated of for $60 \mathrm{~min}$. 

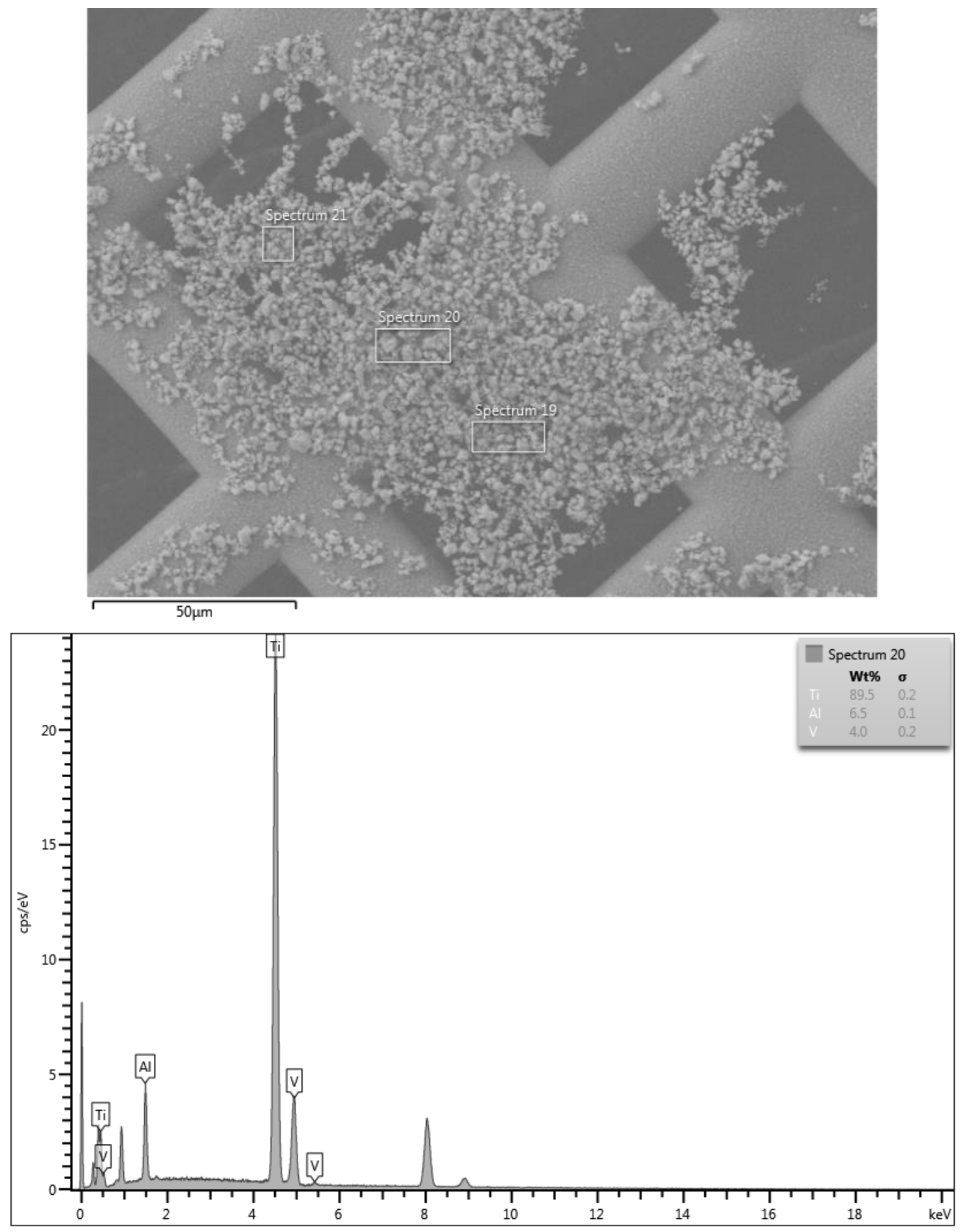

Fig. S4. SEM image of titanium nanoparticles and results of EDS measurements from the specified location confirming that the composition of nanoparticles corresponds to the composition of TiAl6V4 alloy. 


\section{References}

(1) Rak, D.; Sedlák, M. J. Phys. Chem. B 2019, 123, 1365.

(2) Provencher, S. W. Computer Physics Communications 1982, 27, 213. 